\title{
Nota su antichi testi aquilani
}

\author{
Paolo Fasoli
}

Non deve stupire che i primi documenti letterari in volgare abruzzese (la definizione, sia chiaro, riguarda più l'appartenenza territoriale ai tempi correnti, che la tipologia del dialetto) siano apparsi nella città di più recente fondazione della regione, L'Aquila, riedificata, secondo tradizione, dopo le distruzioni manfrediane del 1254. E cio non meraviglierebbe qualora si considerasse che quei documenti sono tutti riferibili alla Compagnia locale dei Disciplinati, fondata in città nel 1266, sembra per volontà diretta di San Bonaventura. Sorto a Perugia tra il 1259 ed il '60, il movimento si diffuse presto in tutta l'Umbria e nelle regioni finitime: all'Aquila, collegata a Perugia dalla via per Rieti, esso prese forma permanente dando origine a più di una confraternita (di San Massimo, di San Leonardo, di San Sisto [De Bartholomaeis, Laude 290]).

Con il nuovo stile di devozione giunse pure l'inclinazione per quei generi indicati tradizionalmente come laudistici, che proprio i Disciplinati avevano ripreso e consegnato a nuove forme e nuova destinazione. L'Aquila fu così uno dei centri della fioritura di teatro (o proto-teatro) religioso che interessò, con esiti omogenei e spesso interdipendenti, l'intera porzione mediana della penisola (il Lazio, l'Umbria intera, l'Abruzzo centro-orientale, Urbino, Siena, Orvieto), donde poi si parti per approdare a nuovi idiomi in regioni più lontane (di Bologna, di Pordenone). La fioritura della lauda drammatica costitù̀ così un fenomeno unico, ed irripetuto almeno fino all'avvio della letteratura canterina nel secondo Trecento, di diffusione di un genere letterario a forti caratteri di omologazione per la destinazione (la pubblica recita) siccome per le forme metriche, per la matrice religiosa e culturale, nonché per il canone tematico (essenzialmente legato al procedere dell'anno liturgico); caratteri che non vennero meno neanche quando il genere si trasfuse in ambito domenicano (e all'Aquila vi furono i Disciplinati di San Tommaso).

Ad agevolare la diffusione del genere contribui, certamente, anche il fatto che essa si irradio a partire da una zona mediana, di transizione tra i dialetti, con notevoli elementi di contiguità (basti, ancor'oggi, vedere la distribuzione delle isoglosse nel territorio umbro) tanto con le parlate delle parti più interne della Toscana centro-meridionale (senese e aretino) quanto con alcune delle marchigiane (Urbino), e con le abruzzesi di carattere linguistico non meridio- 
nale (aquilano). In questo modo, sulla base di affinità linguistiche, si può rendere comprensibile perché, su una vasta area, un testo poté stabilirsi in una sorta di koiné stilistico-formale: gli stessi stilemi, talvolta lo stesso apparato metrico e rimario, potevano trascendere senza variazioni e necessità di adattamento da una parlata all'altra. La lauda divenne dunque uno strumento comunicativo di vigore e compattezza inediti, e ciò poté permettere, forse, che anche da aree laterali, come appunto l'aquilana, si originassero innovazioni (ad esempio nel tipo strofico) destinate a produrre altrove frutti ulteriori.

La prima fondamentale raccolta della lirica e della drammatica dei Disciplinati aquilani sembra essere quella trasmessa dal codice XIII, D, 59 della Biblioteca Nazionale di Napoli proveniente dal convento di San Bernardino da Siena all'Aquila. Si tratta di un codice autografo, di mano di tale Pietro di Nicola, databile approssimativamente alla fine del secolo XV.' Nelle parole di De Bartholomaeis, esso sarebbe "una sorta di Laudario de' Laudari" (Teatro 346), che riunirebbe, contaminandole, varie raccolte, in abdicazione alla linearità tipica dei laudari perugini basati sulla decorrenza dell'anno liturgico. Nel libro copiato da Pietro (che non necessariamente ne fu il compilatore), si trovano infatti sovente, come si vedrà per l'Annunciazione, più laude per una stessa ricorrenza, e vi sono mischiate laude temporali e santoriali. Tra i suoi cinquattotto componimenti devono poi ravvisarsi opere di una certa eccentricità formale o tematica rispetto al canone tradizionale. È il caso della Leggenda di Santa Caterina di Buccio di Ranallo, unico compinimento datato (1330), che dimostra il precoce accostamento degli autori riferentisi ai Disciplinati aquilani ai modelli drammatico-agiografici che si imporranno più tardi, soprattutto a Firenze. D'altra parte, la stessa figura di Buccio, autore preminente del Trecento aquilano, può emblematizzare il carattere tutto o religioso o civile (si pensi alla Cronaca rimata) che la letteratura aquilana dei primi secoli andava manifestando, respingendo quei generi profani e quegli influssi di tipo provenzale, siciliano, stilnovista e poi petrarchista che tutti insieme troveranno alla fine del Quattrocento in un altro aquilano, Serafino, un grande accoglitore, rifacitore e divulgatore.

Altra composizione di estremo interesse presente nel codice è Lo lamento della Dompna, ${ }^{2}$ che l'editore considera, connotando il giudizio in termini negativi, una specie di monstrum formato contaminando due laude, una perugina, l'altra aquilana (De Bartholomaeis, Origini 295), e che invece a me sembra interessante proprio per la preferenza accordata al metro più autoctono tra $\mathrm{i}$ due disponibili nei modelli, ovvero la quartina di doppi quinari monorimi, tipo strofico che si apparenta a quelle quartine di alessandrini, pure monorimi, che sono il verso "ufficiale" della cronachistica aquilana, e che a lungo resistettero all'importazione dell'ottava.

Un documento anch'esso eccentrico rispetto alla tipologia laudistica è il Detto dell' Inferno, componimento difficilmente databile, sorta di contrasto tra 
un vivo ed un morto rinviante tematicamente alla Visio Sancti Pauli, e testualmente all'Elucidario di Onorio di Autun, ovvero a quella serie di testi in cui comincia a intravedersi un 'idea purgatoriale avanti la lettera: si comprende lo stupore di De Bartholomaeis di fronte all'intervenire di riferimenti al Purgatorio in un'imitazione da Onorio, stupore che oggi le ricerche sui testi e la cultura che consentirono la naissance del secondo regno rendono motivato.

Il fondo più antico della raccolta del ms. di Napoli, secondo De Bartholomaeis, sarebbe costituito dalle laude drammatiche di impianto più tradizionale. Tra esse, quattro avrebbero a vario titolo precise ascendenze perugine. Per tre, "l'origine perugina si appalesa manifesta sia nella struttura metrica, sia nelle dimensioni. sia nel genere, sia nello stato di conservazione" (Origini 294). Un'altra, invece, quella dell'Annunciazione (Anunptiatio Sancte Marie) ha più documentabili origini umbre. Inoltre, "tra le umbre, essa è sicuramente una delle più antiche, visto che persino i laudari perugini la danno in redazioni variamente rimaneggiate. Del resto, anche se non la ritrovassimo in quelle raccolte e in quello stato, a dimostrarne l'antichità basterebbe l'estremo scadimento della versione aquilana, ove parecchie stanze persero de' versi e parecchi versi la rima" (ibid.).

Sull'"estremo scadimento" della versione aquilana tornerò tra breve. Ora importa identificare alcuni tra i precedenti umbri cui allude De Bartholomaeis.

Di uno di questi, una lauda perugina, lo stesso studioso fornisce un'edizione nel volume 1 della sua raccolta di Laude drammatiche e rappresentazioni sacre (98-102). Trasmessa in quel Libro de Laode forse già finito al principio del Trecento (De Bartholomaeis, Origini 221), essa consta di 17 strofe per un totale di 116 versi. Delle strofe, 12 sono di 8 versi, 5 di 4 . I versi alternano misure più brevi (settenari, essenzialmente, più qualche ottonario, o novenario difficilmente rettificabile) a endecasillabi più o meno regolari. Se la struttura lascia ancora intravvedere l'ascendenza ballatistica, è tuttavia assente la ripresa, e così pure la rima dell'ultimo verso delle varie strofe non è costante, soprattutto nelle ultime, mentre nelle prime, ma forse solo per inerzialita grafica, appare più come assonanza, alter-nandosi -ate ed -ade. I locutori sono quattro: l'Angelo, Dio Padre, il Figlio, Maria. Il dramma comincia con il lamento dell'Angelo alla Trinità sulla situazione miserevole del genere umano. Segue un dialogo tra il Padre e il Figlio in cui si decide la strategia di salvazione. Concludono l'annuncio a Maria e il dialogo di essa con Gabriele.

Più breve è la lauda dell'Annunciazione che si legge nella raccolta dei Disciplinati di Gubbio, i cui testi, trasmessi dal codice quattrocentesco della Compagnia dei Bianchi di Santa Maria del Mercato del centro umbro, ebbero un'edizione completa per opera di G. Mazzatinti (171-73). La lauda consta di 52 versi divisi in 8 strofe, le prime 3 di 4 versi, le altre di 8 , secondo la stessa alternanza di misure di versi della versione perugina. Essa in pratica contiene lo stesso testo della perugina a partire dall'arrivo di Gabriele al cospetto di 
Maria. Dopo la quinta strofa, però, i testi divergono e l'eugubino presenta una sorta di riassunto, per bocca dell'Angelo, dell'antefatto trinitario, della decisione della redenzione e della resurrezione.

La versione aquilana, invece, è la più lunga. Essa consta di 173 versi, divisi in 24 strofe. Di esse, 14 hanno 8 versi, 9 ne hanno 6, una 7. Per quest'ultima (s. 17), più che alla caduta di un verso, penserei alla geminazione a distanza, forse dovuta ad errore, del v. 2 nel v. 5 ("e non para impossibile / ... / perciò non è impossibile"). Infatti, la misura dei versi (ottonari per lo più, ma anche settenari e novenari) è la stessa delle altre strofe di 6 versi, secondo il modello tipico della lauda passionale avanzata mentre quelli di 8 continuano a presentare l'alternanza tra settenari ed endecasillabi. I punti di contatto con le versioni umbre si limitano alle strofe 5,6 e 7, corrispondenti alle strofe $10,11,12$ del perugino e alle strofe 2, 3, 4 dell'eugubino. Dunque, vi è coincidenza per 3 strofe in tutto. La lauda aquilana comincia con Maria che chiede a Dio Padre chi sia la donna "che deve incarnare". Manca il conciliabolo tra Dio, il Figlio e l'Angelo, mentre si introduce il personaggio della cognata Elisabetta, solo evocata per exemplum nelle versioni umbre.

Per quel che riguarda l'aspetto linguistico dei volgari utilizzati nelle tre versioni, noterei subito che nel testo di Gubbio la coloritura umbra è più evidente nel vocalismo che non nel consonantismo, essendo in quest'ultimo oscurata da abitudini grafiche fortemente conservative, soprattutto riguardo agli esiti dei nessi consonantici latini (v. 2 benedecta; v. 3 electa; v. 6 promixione; v. 8 benedictione; v. 10 nunptiare; v. 24 facta; v. 35 desfacto; v. 42 desdecta).

Sul piano vocalico, l'elemento di identificazione umbra più appariscente è senza dubbio la pressoché regolare presenza di e in posizione protonica iniziale e non, nei casi dove in toscano invece è presente la i (v. 11 emcarnare; v. 22 vergenetade; v. 23 benegnamente; v. 36 desfacto; v. 42 desdecta) (Rohlfs $\S$ 130).

Notevole è anche la presenza di e tonica in vento (v. 36), esito normale, fuori dell'area fiorentina, anche in Toscana, oltreché in Umbria, nel caso in cui in sia seguito da palatale: evidentemente il participio si è qui assimilato per analogia al resto della coniugazione.

Se la versione eugubina è, come si è detto, molto conservativa dal punto di vista della grafia delle consonanti, quella perugina invece presenta esiti indubbiamente caratteristici: tra questi, ad esempio, pate (vv. 45, 53, 70,84) e mate (v. 44), spiegabili come esiti di una riduzione del gruppo -tr-, o, secondo Rohlfs, come "antichi nominativi (vocativi)" (\$ 260) comunque tipici dell'umbro antico tanto quanto del laziale meridionale anche moderno. A questo riguardo, crederei che la parola meta al v. 52 ("essere io serva de sì alta meta") sia un refuso tipografico dell'edizione De Bartholomaeis, dove dovrebbe leggersi mate, o al limite, mete, qualora vi occorresse il fenomeno tipico del perugino della chiusura di $a$ tonica in sillaba libera (Moretti 30); fenomeno che 
tuttavia non ha altro riscontro in questi testi. Sempre nel testo perugino, è evidentissima la presenza di - $e$ paragogica in vocaboli delle più diverse classi grammaticali, sempre in dittongo con una $i$ semiconsonante primaria o rimpiazzante una consonante lenita (vk 6, 35 cuie; v. 18 noie; v. 63 faie; v. 72 haie; v. 74 maie; v. 21 deie, e cf. v. 48 dei; v. 23 deie).

Nella serie pronominale abbondano ovviamente i deittici, trattandosi di un disorso drammatico. Qui le differenze tra il testo aquilano e quello perugino sono interessanti. Nella lauda umbra troviamo quisto (vv. $8,38,88$ ), quil (in correlazione: vv. 83, 97), ma anche quista (v. 56) e quilla (v. 95). Secondo Moretti, il "pareggiamento vocalico" tra maschile e femminile "escluderebbe l'origine metafonetica nella serie di pronomi dimostrativi" (31 n. 18). Secondo altre opinioni, riportate pure da Moretti (ibid.), si tratterebbe di semplice adeguamento del femminile al maschile metafonetico, privilegiando dunque il tipo non toscano. Il testo aquilano, invece, presenta la metafonia da $-U$ nel maschile (vv. 46, 113 quillo), ma non ovunque (v. 140 quello; vv. 147, 149 questo), mentre per il femminile non c'è, come era prevedibile, nessun adeguamento. Altrove, la metafonia (da -i) si accompagna a fenomeni consonantici: è il caso di bui (vv. 29,39,42), dove la grafia $b$-doveva forse rendere, come in testi più antichi (lo stesso Ritmo cassinese) un suono bilabiale, che qui reputerei favorito anche dalla $u$ metafonizzata(Rohlfs $\S 167$ ).

Un altro fenomeno di vocalismo tipico dell'aquilano (la conservazione di $-u$ delle finali in - US di seconda declinazione primaria o originaria della quarta) è qui attestato ai vv. 106 e 108 (Spiritu Sanctu).

Sempre nel testo aquilano, per i possessivi, prevalgono le forme autoctone (vv. 5, 14, 153 soa; vv. 16, 60, 96, 106 toa; v. 19 tou; v. 54 sou; presenti anche le varianti tea, vv. 31 e 116; sea, v. 120), mentre esigenze di rima impongono tia al v. 133, con adozione di una forma che peraltro è tipicamente umbra.

Per quel che attiene al lessico, la lauda aquilana presenta per due volte (vv. 134, 157) la voce quinata per cognata. In questa forma, la parola è attestata in tutta la produzione laudistica aquilana solo in un altro componimento, pur esso un' Annunciazione, e che è trasmesso nello stesso manoscritto dell'opera in esame. Si tratta di una forma che non sono riuscito a rintracciare altrove, ed assente nei maggiori dizionari delle parlate d'Abruzzo. Il dizionario di Giammarco riporta, come forma più simile, un cunat plurale. Quello di Finamore cunate, cunete, cunuete, cunuate, per altro fuori area (ad Atri). L'esito in da -gn-(non palatalizzato) è abbastanza comune nell'Italia centrale e meridionale (napoletano cainato, o caienato; a Campobasso kajenat) (Rohlfs § 259). Cio che qui stupisce è non solo e non tanto il passaggio di $o$ ad $u$ anziché l'apertura in $a$ a seguito dello sviluppo di $i$, ma anche il fatto che, per ragioni metriche, la $u$ verrebbe ad essere semivocalica, in dittongo con $i$, come pure la scelta della notazione labiovelare da parte dell'editore sembra segnalare (e si presume che si trovi pure nel ms., che per altro non sembra mostrare, se l'edizione è conservativa come si presenta, particolari anomalie' grafiche). 
Nel testo perugino, invece, l'anomalia più evidente riguarda un passato remoto di terza persona singolare del verbo concepire, reso, al v. 92, con conceiò, laddove il testo eugubino, allo stesso luogo, ha un più regolare concepecte (v. 49). In entrambi i casi, il perfetto originariamente forte (cepi) viene normalizzato in debole, ma nel secondo caso viene adottata l'uscita accentata in -o della coniugazione in $a$. Così, la consonante occlusiva labiale si sarebbe dileguata, dando luogo ad una semiconsonante.

Se per la cronologia assoluta di questi testi bisogna ancora fidarsi dell'impostazione della scuola storica, per quella relativa condivido in pieno il giudizio di De Bartholomaeis sulla seriorità della versione aquilana. Tuttavia, essa per chi scrive, si inferisce da altro tipo di indizi, e soprattutto, dal processo di rielaborazione, dilatazione, accentuazione drammatica che vi si presenta, e non da un presunto "scadimento", che a me non pare si possa ravvisare. Forse la presenza di alcuni "arcaismi" doveva turbare la visione sinceramente evoluzionistica che De Bartholomaeis aveva del metro delle laude. Secondo lui, infatti, un unico processo aveva portato dall'espunzione della ripresa dalla forma ballatistica tradizionale di tipo pasquale e jacoponico all'abolizione della rima finale unica e poi al passaggio dalla sestina di ottonari a quella di endecasillabi e infine alla "gloriosa ottava" (Teatro xv-xvi). Se quest'ultimo passaggio sembra ora trovare autorevoli sostenitori tra coloro che si occupano della genesi dell'ottava toscana, la visione di De Bartholomaeis si presta però ad essere smentita, nel suo evoluzionismo, dalla prova dei fatti. Così, ad esempio, la Leggenna de Santo Tomascio, testo avanzatissimo se non altro sul piano scenico, adopra un multimetro fatto di tipi autoctoni (versi doppi) e umbro-toscani (ottonari, settenari, endecasillabi in varia misura strofica).

Nell'Annunciazione aquilana, invece, mi sembra che nel processo rielaborativo convivano la tensione al nuovo ed il richiamo alla tradizione. Così, se la misura strofica è più regolare che nella lauda perugina, e se in essa troviamo strofe di 8 versi frammiste a strofe di 6 versi anziché a quartine (dunque vi appaiono le sestine della forma passionale compiuta), d'altro lato però compare pure il richiamo alla chiusura ballatistica con l'unicità della rima finale di ogni strofa, unicità peraltro osservata in modo molto più costante che nei componimenti umbri. Dove poi sia lo "scadimento" ravvisato da De Bartholomaeis io non saprei dire, né mi sembra che davvero dei versi o delle rime siano caduti, né è più forte l'anisosillabismo che nei due testi umbri, né la condizione generale del testo e, a men che non sia stato troppo restaurato, tanto pietosa.

Tra i richiami alla tradizione includerei pure l'inserzione dei versi o emistichi in latino liturgico, sconosciuta alle versioni umbre,e che sembra quasi voler sorprendere la lauda nella sua origine responsoriale, sottolineandone la natura di mutante, di tropo all'interno del testo rituale.

D'altro canto, invece, l'aumentato numero dei personaggi va verso la direzione del dramma più composito, sul tipo della Leggenna. 
Altre versioni impongono al dramma un cappello lirico-narrativo che sembra puntare al massimo sulla mistione dei generi. Ci troviamo forse in una di quelle fasi intermedie, in quegli incroci di generi letterari che nel Medioevo possiamo constatare in reliquie spesso fuori contesto. Del resto, che tipo di actio supponevano questi testi?

Infine, mi sembra importante notare come la lauda aquilana da qui esaminata vada poi a farsi parte di un ulteriore processo elaborativo di area abruzzese. Essa, nella forma qui discussa, si diffonde in altre zone, dialettologicamente allotrie: la si rinviene ad esempio in un codice di Capestrano proveniente da un convento francescano di Penne; linguisticamente, invero, assai poco mutata (per quanto consenta di appurare il diverso stile grafico). Qui, pero, essa presenta, come già si è accennato, una sorta di prologo che, iniziato con un'invocazione alla Vergine (tratto che poi sarà caratteristico di ogni narrativa, anche profana), procede con una narrazione degli antefatti secondo il modo in cui essi erano presentati all'inizio delle versioni umbre (e si tratta delle strofe non riprodotte nella versione aquilana). Il cappello narrativo non è isolato, ma continua con una sorta di interruzione didascalica tra la prima e la seconda strofa.

$\grave{E}$ interessante notare come lo stesso prologo si trovi in una versione identica, ed unito ad un'altra lauda d'annunciazione (testo che nulla condivide con le redazioni umbro-aquilane), contenuta nello stesso codice dov'è la versione aquilana in esame (De Bartholomaeis, Teatro 279-81). Viene così a crearsi una sorta di microtradizione parallela che sembra rendersi disponibile quando la lauda forse già perde udienza, o non più soddisfa previsioni di successo, per l'accedere alle scene di testi più complessi, del tipo della Leggenna de Santo Tomascio.

Hunter College of the City University of New York

\section{NOTE}

1 Si veda la descrizione in De Bartholomaeis, Teatro 345-49.

2 Per l'edizione, cfr. De Bartholomaeis, Teatro 16-20.

\section{OPERE CITATE}

La leggenna de Santo Tomascio. Ed. Fabrizio Beggiato. Cortona: Centro di studi sulle origini del teatro italiano, 1969.

Beggiato, Fabrizio. "Contributi alle edizioni della Leggenna de Santo Tomascio". Abruozo 9 (1971): 269-75.

De Barholomaeis, Vincenzo. Teatro abruzzese del Medioevo. Bologna: Zanichelli, 1924.

\section{Laudi e rappresentazioni sacre. 3 voll. Firenze: Le Monnier, 1943.}

Origini della poesia drammatica italiana. Torino: SEI, $1952^{2}$.

Finamore, Gennaro. Vocabolario dell' uso abruzzese. Città di Castello: Lapi, 1893.

Giammarco, Emesto. Abruzzo. Pisa: Pacini, 1979. 
Dizionario abruzzese e molisano. Roma: Ateneo, 1968-85.

Storia della cultura e della letteratura abruzzese. Roma: Ateneo, 1969.

Abruzzo dialettale. Pescara: Ferretti, 1973.

Mazzatinti, Giuseppe, "Laude dei Disciplinati di Gubbio". Il Propugnatore 2.1 (1889): 10-60.

Moretti, Giovanni. Umbria. Pisa: Pacini, 1987.

Rohlfs, Gerardt. Grammatica storica della lingua italiana e dei suoi dialetti. 3 voll. Torino, Einaudi: 1968. 